\title{
Retorno en tiempos de crisis: un ejemplo de Ecuador
}

Eleder Piñeiro Aguiar

Los inmigrados y "minoritarios" nos traen de regreso nuestro propio inconsciente, iel cual inhibimos! Al reconocer las economías étnicas bajo la máscara de lo cultural que es el efecto de su rechazo por parte de nuestra historia, contamos con el primer medio para evaluar lo que está en juego.

De Certeau

\section{Introducción}

"No es una crisis, es que ya no te quiero". Esta frase puede sonar a fin de relación sentimental entre dos personas, pero es mucho más que esto. Fue la frase de manifestación utilizada en pancartas y grafitis en el año 2011, cuando diferentes personas comenzaron a pronunciarse ante la situación de precariedad y descontento social generalizado en España. Se estaba fraguando el movimsiento del 15-M —indignados o spanish revolution según otros discursos/medios-. Paralelo a esto, mucha gente se estaba marchando del país, pues la crisis que se inició en torno a 2008 obligaba a buscar alternativas a cientos de miles de personas en edad laboral, principalmente jóvenes. 
Pero la situación no es ni mucho menos exclusiva de España. La crisis global producida —este término no es neutral— ha generado, entre otros aspectos, "extraños llamando a la puerta" (Bauman, 2016). Nos centraremos en ciertas repercusiones que esta crisis ha tenido en la movilidad humana, y en concreto en la categoría del retorno, tomando principalmente como contexto Ecuador en los últimos años.

Según Durand (2006, p. 167), las teorías que han tratado de explicar el proceso del retorno migrante han sido "acercamientos parciales a una realidad compleja y cambiante”. Incluso expone este autor que existe cierto enigma cuando un migrante del denominado primer mundo, tras una estadía más o menos prolongada, decide regresar a su país de origen en el denominado tercer mundo. La visión tradicional del análisis de las migraciones ha supuesto que gente pobre de países pobres elige como destino economías de países ricos, con un énfasis en una visión utilitarista-racionalista propia de la economía clásica y neoclásica. Pero la realidad social ha demostrado que esto no siempre es así y de hecho nunca son las personas más pobres —ni en proporción los países más pobres- los que emigran —o los que más emigran-. Así lo expone Saskia Sassen al analizar las relaciones de la migración con el nacimiento y reproducción del capitalismo y de la conformación de los Estados-nación:

Aunque un Estado careciera de los medios técnicos para controlar sus fronteras, la inmigración era un proceso selectivo: no todo el mundo dejaba áreas pobres para desplazarse a otras más ricas, aunque pudieran llegar a ellas caminando en dos días o una semana. Pero el pasado también nos dice algo sobre las condiciones actuales: puede que obsesionarse con los controles de frontera para regular los flujos migratorios no sea de mucha utilidad (Sassen, 2013, p. 13).

A lo antedicho se puede afirmar también que se ha ido superando una visión excesivamente individualista, centrada en el sujeto migrante, para dar paso a otras teorizaciones que hablan de redes, de cadenas, de transnacionalismo y de factores que van más allá de las típicas visiones push-pull del utilitarismo economicista. Y dentro de esto, las políticas de retorno son uno de los dos grandes grupos de políticas que 
los Estados emprenden para relacionarse e interactuar con sus ciudadanos en el exterior (Moncayo, 2011). En otro grupo estarían las políticas para vincularse con las diásporas, si bien en ocasiones ambas pueden estar conectadas e incluso unas $\mathrm{u}$ otras pueden tener diferentes intensidades dependiendo del momento económico, de las políticas de extranjería supranacionales, de las demandas de mano de obra en ciertos sectores, entre otras. "Al parecer, la nuestra es una era transnacional en la que son pocos los países de origen de emigrantes que no tienen diásporas que están tratando de movilizar, y existen pocas emigraciones en las que no encontramos una acción social consciente de la diáspora.” (Waldinger, 2013, p. 191).

Las políticas de retorno voluntario serían aquellas que brindan asistencia en forma de logística, recursos financieros, apoyos a la movilidad o al emprendimiento, siendo dicha asistencia escogida de manera libre y voluntaria por los migrantes. En este sentido, una taxonomía interesante es la elaborada por Mestries (2013), quien expone que existen dos tipos de retorno: voluntario y forzado. Cada uno de ellos presenta diferentes causas, motivos y resultados. Entre las causas del retorno voluntario separa dos grandes grupos: psicológicas y culturales; y económicas. Como motivos del retorno para estas dos causas - las cuales desde nuestro punto de vista deben ser vistas de forma integral-, Mestries expone que existen motivos afectivos familiares, socioafectivos - redes-, desengaño en cuanto a las expectativas —aquí funcionarían los imaginarios, como por ejemplo el american dream o la Europa desarrollada, primer mundo-, cumplimiento del proyecto - ahorro-, aprendizaje de un oficio nuevo, recortes laborales, alza del costo de vida, mejoras en el país de origen.

Los resultados de todos estos motivos son varios: reunificación, reinserción en la comunidad, autoafirmación, independencia económica, cambio de sector productivo y demás. Se pueden agregar otros como cambio en los roles familiares, renegociación de la identidad propia o familiar, conflictos parentales o intergeneracionales, cambios en las cadenas del cuidado u otros.

Siendo esta una de tantas clasificaciones acerca del retorno, lo que no se puede obviar es que los avances del neoliberalismo, las desregulaciones, la flexibilidad laboral, las condiciones en origen y destino y 
un sinfín de causas y variables más, inciden en las decisiones —nunca libres y casi nunca del todo voluntarias - a la hora de emprender la movilidad hacia otras zonas del planeta. Las tomas de decisiones a la hora de emprender el retorno son tanto o más complejas que las de la primera migración. El retorno es siempre un fenómeno pluricausal en el que se debe tener en cuenta, como recuerda Schramm, "la consideración de las fases anteriores a la decisión de retornar" (2011, p. 242). Este autor analiza tres tipos ideales - a la manera de Weber- de migrantes: constantes, fracasados y buscadores, los cuales tendrán diferentes redes, estrategias y proyectos migratorios. Los primeros tendrán el retorno de una forma más planificada y una vida en destino más relacionada con el ahorro. Los otros dos grupos se verán mucho más condicionados por cuanto no dispondrán de capital, redes, ahorro; tendrán menor autonomía de cara a poder llevar a cabo un retorno y no podrán preparar el retorno de forma adecuada.

Dos de los principales factores que inciden son la situación en el país de origen y la posibilidad de volver a migrar en caso de posible fracaso del proyecto de retorno. A la complejidad de factores se suma que no todos los países llevan una estadística pormenorizada de sus retornados, y no se suelen cruzar datos entre diferentes Estados. Algunos problemas analíticos de la medición del retorno, o su "vaguedad conceptual" (Castillo, 1997, p. 29) tienen que ver con la naturaleza - definición-, la medición, la compatibilidad de fuentes y la cobertura (López de Lera, 2010, p. 16).

Debido a todo esto es más que pertinente profundizar en los análisis del retorno y de los retornados y generar políticas públicas que vayan hacia una mayor integralidad de las fuentes y, por tanto, de las personas en movimiento. Analizar el retorno simplemente como la parte final del periplo migratorio no es suficiente, pues nunca se puede considerar como algo definitivo. De hecho emigrar, inmigrar y retornar son vistos como tres etapas de un mismo proceso (López de Lera, 2010). Desde el campo del transnacionalismo incluso se pueden entender las estrategias y tácticas migrantes como una "diálectica de oposición y resistencia a la lógica económica del capital multinacional" (Guarnizo y Smith, 1998, p. 5). Y si este es por definición cambiante, las resistencias al mismo han de serlo también, por lo cual todo 
proceso migratorio no es algo definitivo como tampoco la estrategia del retorno es algo cerrado y concluyente.

A lo largo de las siguientes páginas se expondrán algunas teorías para comprender la relación entre la crisis financiera y económica global y la vinculación y repercusiones para los sujetos desplazados en situación de retorno. A continuación se verán aspectos culturales-simbólicos en torno a la construcción migrante como categoría para analizar sucintamente cuál es la influencia en ciertos planes de retorno migrante. Se finaliza con un apartado de conclusiones y todo ello desde un punto de vista descriptivo-interpretativo con enfoque basado en la antropología social y cultural, dedicando especial énfasis a las políticas sobre retorno en Ecuador y España en los últimos años, en especial a partir de la crisis de 2008 y los cambios constitucionales en el país andino.

\section{Crisis y desplazamientos globales}

La pobreza, el subdesarrollo y la falta de oportunidades — los legados del Imperio en todas partes- pueden obligar a la gente pobre a emigrar produciendo la disgregación y la dispersión; pero seguimos creyendo de corazón que cada diseminación comporta la promesa de un regreso redentor. Stuart Hall

La frase acuñada por Martin y Teitalbaum (2002) al principio de los 2000, según la cual "no hay nada más permanente que un trabajador temporal” es más pertinente que nunca en el año 2017. Y es que "el tema del retorno de los migrantes internacionales se ha puesto de actualidad con el estallido de la crisis mundial de 2007" (Mestries, 2013, p. 171). Los datos apuntan a que la crisis ha aumentado sustancialmente el número de retornados en todo el planeta (Pajares, 2010, p. 118).

En Ecuador, tras las migraciones de los años sesenta - principalmente motivadas por la crisis de demanda de sombreros de paja toquilla, siendo los lugares de destino Estados Unidos, Canadá y Venezuela—; y las de los años ochenta —encabezadas por sectores rurales de regiones costeras desplazados a Estados Unidos-, se presentaron hacia finales del siglo pasado grandes desplazamientos poblacionales motivados 
por las crisis económicas de los años 98 y 99. En concreto a raíz del denominado "feriado bancario" y la dolarización de la economía en el año 2000, produciéndose la "estampida migratoria" (Ramírez, F. y Ramírez, J., 2005) en este país, fecha en la que se produjo un "giro en la migración ecuatoriana ya que es el periodo que presenta la curva más alta de migración con un saldo migratorio de 175922 ecuatorianos" (Dirección Nacional de Migración de Ecuador, 2006, citado en Cortés, 2009, p. 89). En ese proceso, en el año 2005, la ecuatoriana es la nacionalidad latinoamericana más numerosa en España (Moreno, 2006, p. 122). Desde el año 2000 las remesas se convierten para Ecuador en la segunda fuente de divisas, tras la exportación petrolera (Villamar y Acosta, 2002).

Uno de los destinos principales para la movilidad en esos años fue España, debido a la bonanza de su economía en esa época, la habilidad de hablar el mismo idioma y las facilidades a la hora de conseguir un visado. Así lo atestiguan los 135000 ecuatorianos en España en 2001, según el Ministerio de Interior de ese país. Este visado se obtenía de manera sencilla: "Simplemente la exigencia de empadronarse en el Ayuntamiento al ingresar y eso bastaba para acceder a derechos de salud, regularización y educación" (Padrón, 2015, p. 16). En concreto, el área de la construcción era el que más migración ecuatoriana reclutaba de todos los sectores laborales en el último año previo a la crisis, con casi la cuarta parte de migrantes dedicados a esa sección de la economía (Duque y Genta, 2009).

Pero la situación cambió por completo hacia 2007-2008. Las restricciones para obtener visados aumentaron y la situación en destino empeoró para muchas familias, algunas de las cuales decidieron retornar al país andino. En esta tercera etapa de emigración ecuatoriana, donde se incluye la inmigración de retorno, no solo aumentó significativamente el número de migrantes, sino las categorías de los mismos: migrantes en tránsito, refugiados — principalmente desde Colombia- y, por supuesto, aumentó el retorno, en especial en los últimos años debido a las crisis globales y que, en concreto, afectaron a ciudadanos ecuatorianos que estaban residiendo principalmente en Estados Unidos, Italia y España. Pérdida de viviendas, deudas adquiridas con los bancos, masivos despidos principalmente en el sector 
de la construcción y ajustes de las leyes migratorias, haciéndolas más restrictivas en España, son algunas de las principales causas del retorno, en aumento desde 2009. En el siguiente cuadro se pueden observar las entradas de ecuatorianos a Ecuador, lo cual contrasta con los números que aporta el Ministerio de Trabajo de España en cuanto a retorno voluntario. Se debe recordar que dicho momento se inserta en un contexto más amplio, en el cual la Unión Europea aprobó la directiva de retorno (18 de junio de 2008), la cual fue denominada “directiva de la vergüenza" por varios colectivos migrantes, varias ONG, Mercosur, Comunidad Andina de Naciones, entre otras. El nombre de directiva de la vergüenza se debe a una Carta Abierta firmada por el presidente boliviano Evo Morales, la cual concluía con un saludo solidario "a todos nuestros clandestinos".

La Directiva ha sido fuertemente criticada, especialmente por parte de los países de origen de los inmigrantes que viven en Europa, debido a la lógica securitizadora que subyace detrás del instrumento, que se basa en elementos restrictivos y de coerción, descuida los derechos de los inmigrantes y disminuye sus garantías jurídicas (Moncayo, 2013, negrita en el original).

Según Moncayo (2013) dicha Directiva sentó un precedente por cuanto refuerza la idea de que los primeros en sobrar en una crisis son los extranjeros, sobre los cuales hay que elaborar políticas de vigilancia y control, todo dentro de unas lógicas securitarias, siendo la gestión del retorno un aspecto clave tanto para la continuidad de discursos "nosotros-ellos", para aliviar supuestamente las tensiones económicas de la crisis. “Ellos siempre son demasiados. 'Ellos' son los tipos de los que debería haber menos o, mejor aún, absolutamente ninguno. Y nosotros nunca somos suficientes, 'nosotros somos la gente que tendría que abundar más'” (Bauman, 2005, p. 51, comillas y cursivas en el original). A esto, Moreno (2006, p. 124) le denomina fundamentalismo cultural "que señala la llegada de inmigrantes de países pobres como un problema, una invasión, una avalancha que debe ser controlada y regulada con leyes de extranjería cada vez más estrictas”. 
Cuadro 1. Comparación entradas a Ecuador y programa retorno voluntario

\begin{tabular}{|c|c|c|}
\hline Año & $\begin{array}{c}\text { Entradas ecuatorianas } \\
\text { al Ecuador }\end{array}$ & $\begin{array}{c}\text { Programa retorno voluntario } \\
\text { de atención social }\end{array}$ \\
\hline 2009 & 820292 & 258 \\
\hline 2010 & 893408 & 225 \\
\hline 2011 & 1027543 & 275 \\
\hline 2012 & 1025310 & 202 \\
\hline 2013 & 1143116 & 399 \\
\hline 2014 & 1269675 & 294 \\
\hline
\end{tabular}

Fuente: elaboración propia a partir de Inec (2014). Anuario de estadísticas de entradas y salidas internacionales 2014 y del Ministerio de Empleo y Seguridad Social (2015)

Como se puede observar, pese a que ha ido en aumento el número de entradas de ecuatorianos a su país de origen — recordamos que se trata de entradas, no de migrantes; esto es, una misma persona puede aparecer varias veces-, si se le compara con el número de personas que se han acogido a los planes que el gobierno de España ha elaborado para fomentar el retorno de extranjeros, en este caso ecuatorianos, las cifras son muy bajas. El eje central de dichos planes para incentivar el retorno por parte del gobierno de España fue entregar conjuntamente la prestación por desempleo, bajo la condición de no regresar al país europeo al menos por tres años, y de irse de España en un plazo inferior a un mes. En caso de que una vez se hayan cumplido los tres años en Ecuador y se pretenda regresar a España, las condiciones serán como residentes temporales o permanentes, es decir, se pierden los derechos adquiridos de cara a una potencial nacionalidad o ciudadanía en el periodo de estancia anterior.

Además de esto, este Plan de Retorno Voluntario está centrado principalmente en que el migrante retornado genere algún tipo de emprendimiento en origen y en general estos planes "utilizan la idea de un retorno 'voluntario' que, en realidad, no lo es, pues las personas que se acogen a dichos programas se encuentran en situaciones extremas $y$, por tanto, no tienen otra alternativa que la de retornar a su país de origen" (Moncayo, 2011, p. 3). 
Surge la pregunta acerca de si no existe discriminación de género, pues una gran parte de mujeres ecuatorianas trabaja en el sector informal de la economía principalmente dedicadas al cuidado y al servicio doméstico, por tanto, no serían perceptibles de ser objeto de dicha prestación, reforzando todavía más la vulnerabilidad en la que se encuentran las mujeres migrantes (Moreno, 2006, p. 121). Pagnotta (2005) se refiere a este tipo de trabajos como "condición de servidumbre posmoderna”, por cuanto las mujeres deben someterse a las disponibilidades del empleador, a no tener rutinas, a no estar regularizadas, a someterse a la máxima flexibilidad laboral, a no poder disponer de vacaciones ni días libres.

En cualquier caso, tal como manifiestan las cifras, el programa ha estado lejos de ser exitoso. En estos planes, Ecuador está por debajo de países como Argentina, que para el total del periodo acogió a 1925 personas entre 2009 y 2014, el cual es encabezado por Bolivia (3250). Brasil con 1913 personas que solicitaron y obtuvieron el plan es el tercer país, un puesto por encima de Ecuador, que presenta un total de 1653 retornos.

Pero no solo España generó programas de retorno voluntario, como el antedicho "Plan de Retorno Voluntario", generado en 2008. En Ecuador, en ese mismo año, se crearon los programas "Bienvenidos a Casa" y "Fondo Cucayo", dentro de convenios bilaterales con España.

Según Araujo y Eguiguren las características que los países andinos tienen a la hora de relacionarse con sus diásporas y de confeccionar políticas públicas hacia sus nacionales en el extranjero se acoge a cuatro objetivos:

[...] una perspectiva de seguridad que prevalece, de manera relativamente visible durante los últimos 20 años; una perspectiva donde prima la defensa de los derechos humanos de los migrantes; un modo de gestión que vincula la migración con las posibilidades de desarrollo de los países de origen; y, finalmente, una tendencia que desarrollan los estados en relación con su población emigrada de forma exclusiva con el fin de construir y mantener vínculos con las respectivas diásporas, a través de mecanismos económicos, políticos y culturales (Araujo y Eguiguren, 2009, pp. 2-3). 
En el caso de Ecuador, las políticas de retorno han tenido un discurso de devolver la dignidad a los compatriotas expulsados debido a las consecuencias que el neoliberalismo tuvo en sus vidas, las cuales, según el expresidente Rafael Correa, fueron "nefastas políticas". Así lo expuso en visita oficial a España en 2007, en donde en un acto en asociaciones migrantes en Madrid, previo al inicio de la constituyente de la que saldría la Constitución de Montecristi (2008), afirmó hacia los migrantes que "son ustedes los que sostienen al país con sus remesas" y "al Ecuador no lo mantienen los ricos, lo mantienen ustedes, los pobres, los migrantes”. En este sentido, la Constitución expone la no discriminación, entre otros motivos, por condición migratoria, reconociendo "el derecho a transitar libremente por el territorio nacional y a escoger su residencia" (art. 66).

Diez años después, en 2017, la Ley de Movilidad Humana reconoce la "ciudadanía universal y la libre movilidad", lo cual es un hito en el marco normativo a nivel internacional. Entre las finalidades de dicha ley figura "establecer lineamientos sobre las políticas públicas que el Estado desarrollará para alcanzar el retorno planificado de las personas ecuatorianas desde el Exterior”, colocando en su artículo 24 las atribuciones para la protección, entre las que figuran difundir iniciativas y programas de retorno.

Aún así, esta ley expone únicamente dos tipos de retorno: voluntario y forzado (artículo 26), denominando al primero como aquel que es desarrollado por "la persona que retorna al país de manera libre y voluntaria para establecerse en Ecuador". Como se ha expuesto, dicho establecimiento puede ser definitivo para cierto conteo estadístico en cierto periodo, pero no se puede comprender nunca como cerrado. Y, por otra parte, la libertad y voluntariedad a la hora de decidirse por el retorno queda en entredicho dadas los múltiples condicionantes estructurales, relacionales y culturales en los que están insertos los migrantes.

En Ecuador, el plan "Bienvenidos a Casa" ha sido el principal estandarte de las políticas públicas hacia los retornados, las cuales conciben el retorno en un sentido amplio por cuanto consideran que no se trata tanto de un simple regreso físico sino de recuperar capacidades políticas, culturales, sociales y económicas, en la línea de lo antes 
expuesto de "devolver la dignidad". En cualquier caso, el énfasis está nuevamente en el desarrollo y en el emprendimiento locales.

Las políticas de retorno que implementan tanto los países de origen como los de destino constituyen mecanismos de gobernabilidad migratoria que responden a los intereses de los Estados. En este sentido, por una parte, se evidencia que la mayoría de estos instrumentos de política privilegian a los migrantes que vuelven de forma voluntaria y han acumulado recursos - tangibles e intangibles- por sobre aquellos que regresan de manera forzada y tienen menos recursos, con lo cual sobresale un enfoque económico en detrimento de los derechos de los retornados (Moncayo, 2011, p. 9).

Si al principio del texto se veía que las teorías economicistas en el análisis de las migraciones han sido superadas por nuevas perspectivas que consideran otros factores, no sucede lo mismo con las políticas migratorias, las cuales siguen considerando un enfoque estatalista-economicista-utilitarista-individualista-securitario como axioma principal para entender y gestionar la movilidad humana. A continuación se analizará qué se puede agregar a este enfoque desde la ciencia social.

\title{
Imaginarios de y para la movilidad humana
}

\author{
Lo que para algunos aparece como globalización, \\ es localización para otros; \\ lo que para algunos es la señal de una nueva libertad \\ cae sobre muchos más como un hado cruel e inesperado. \\ La movilidad asciende al primer nivel entre los valores codiciados; \\ la libertad de movimientos, una mercancía siempre escasa \\ $y$ distribuida de manera desigual, \\ se convierte rápidamente en el factor de estratificación \\ en nuestra época moderna tardía o posmoderna. \\ Bauman \\ Para entender algunas de las motivaciones del retorno, es posible es- \\ cudriñar en el campo de la construcción cultural de la realidad, pues
}


existe, además del viaje material, un viaje simbólico. Qué se imagina el migrante a la hora de optar por retornar es uno de los aspectos más importantes para comprender el fenómeno, pero también uno de los más complejos de analizar. Las particularidades de cada uno entran en juego, y tanto como los aspectos estructurales de su situación existen causas que tienen que ver con el deseo, con el espíritu de aventura, con los sentimientos, con las sensaciones, con los lazos afectivos, entre otros.

Para estos imaginarios dos aspectos son clave: las tecnologías de la información y comunicación, gracias a las cuales el contacto, pese a no ser físico, es mucho más constante, cada vez más económico, en tiempo real y con alto crecimiento en cuanto a usuarios e interacciones: "Cuando la distancia 'pierde su significado', lo mismo sucede con las localidades, separadas por distancias. Pero augura la libertad de crear significados para algunos” (Bauman, 1999, p. 29). Y por otra parte, son clave también las relaciones del Estado con sus denominadas diásporas, lo cual puede ser muy útil para producir o reforzar sentimientos de comunidad y lealtades hacia el país de origen. Tan solo utilizando estos componentes, y las teorías de redes y transnacionalismo migratorio, se puede desmontar el excesivo énfasis en el individuo y en el Estado como agentes principales de la migración. Algunos autores denominan "remesas sociales" o conjunto de valores a este tipo de componentes culturales y simbólicos que los migrantes utilizan en la difusión de ideas, sentimientos, bienes, productos, discursos: "Estilos de vida, innovaciones, pautas de comportamiento y capital social que discurren entre las comunidades de destino y origen" (Levitt, 2001, citado en Parella y Cavalcanti, 2006, p. 250). Dichas remesas sociales transforman sociedades, no solo en el plano micro sino incluso en un plano social y político.

Dicho transnacionalismo ha sido definido como "actividades transfronterizas recurrentes, duraderas, significativas, forjadas por grupos basados en dos o más países. Estas actividades pueden ser económicas, políticas, sociales y culturales" (Portes, Guarnizo y Landolt, 2003). El campo de la movilidad humana es mucho más amplio de lo que supone el enfoque nacionalista/individualista; y el análisis del retorno así lo confirma. Transnacionalismo y desarraigo son dos caras de la misma moneda. 
Es un hecho generalizado que la mayoría de los emigrantes parten del terruño pensando en volver. Pero para muchos el regreso efectivo a la tierra de origen se vuelve en la práctica un proyecto cada vez más lejano. No obstante, por más que pasen y pasen los años, siempre queda el gusanillo dentro, la esperanza de volver en la época del retiro, de retornar cuando cambie la situación política, cuando mejoren las oportunidades laborales o económicas (Durand, 2006, p. 174).

En el caso concreto de las diásporas se debe analizar la identidad, en cómo una persona o grupo se identifica y es identificado por otros. Esto será clave a la hora de comprender, por ejemplo, la adaptabilidad al medio, el reconocimiento hacia el país de origen y destino, el sentirse integrados o excluidos, la reconfiguración de la identidad: aprender un idioma, cambiar costumbres, reelaborar tradiciones, cambiar la dieta, estar atentos a nuevas modas, hábitos, formas de convivencia, horarios, entre otros. Todos estos aspectos serán puestos en juego a la hora de la decisión de retornar.

Las prácticas migrantes "habitan nuestros terrenos epistemológicos” (De Certeau, 1995a, p. 197): la economía inmigrante hace entrar en nuestro campo esta economía táctica. Los procedimientos ayer expulsados se implantan en el interior de lo que hacemos del espacio donde habitamos. Es a estas prácticas, a su arte, a sus combinaciones, a las que hay que otorgar el derecho y los medios para ejercerse en las maneras de habitar, soñar, aprender (De Certeau, 1995a, p. 219).

Uno de esos sueños será el del retorno, el cual en muchas ocasiones se convierte en mito por cuanto el regreso se hace imposible. "La mayoría de los inmigrantes responden afirmativamente cuando se les pregunta si piensan volver a su país de origen, la idea del retorno va con ellos cuando se van de su país, pero esa aspiración se va alterando con el paso del tiempo" (López de Lera, 2010, p. 13). Las expectativas generadas en origen, en ocasiones se desmontan en destino, algo que asimismo repercute en el retorno real o potencial a emprender después: "Los migrantes comprueban que existe una brecha de gran magnitud 
entre la representación de las condiciones de trabajo que traen desde el lugar de origen y el alto grado de precariedad laboral a que se enfrentan a su llegada" (Pedone, 2013, pp. 62-63).

Entre los motivos de una negativa a retornar están el alto costo de los pasajes e incluso la imposibilidad de generar nuevos emprendimientos en origen; unas situaciones inestables a nivel estructural en los países desde los que inició la primera migración; nuevos lazos afectivos en el país actual; búsqueda de obtención de ciudadanía y nacionalidad, lo cual retrasa el posible retorno y deviene en asentamiento definitivo, entre otras. Quizá, más que analizar por qué la gente migra o retorna, tendríamos que detenernos a analizar por qué la gente se asienta y no regresa a las raíces: "Al adoptar los patrones locales de consumo, conducta y preferencias, los migrantes y sus descendientes también se vuelven cada vez más diferentes de quienes se quedaron en casa" (Waldinger, 2013, p. 192).

Existen asimismo otros imaginarios, en negativo, que se relacionana con las ideas que los nacionales-autóctonos tienen sobre los migrantes y que pueden incluso reproducirse en el caso del retorno, pese a que no existan diferencias administrativas en cuanto a las identidades de los retornados y de los nacionales. Los nacionalismos que se oponen a la llegada e integración de los migrantes conlleva discursos ciertamente antiinmigrantes, racializados, xenófobos y estereotipados que incluso forman parte de los programas de algunos partidos, sobre todo en Estados Unidos y Europa, lo cual incide directamente en el tipo de políticas públicas acerca de la movilidad humana, las cuales son necesariamente tenidas en cuenta a la hora de emprender tanto la migración como el retorno. "En la actualidad, los inmigrantes aparecen como forasteros amenazadores que llaman a las puertas, las echan abajo o las cruzan a hurtadillas para entrar en sociedades que son más ricas que aquellas de las que proceden" (Sassen, 2013, p. 29).

Las trabas y costos administrativos a los que se enfrentan los migrantes y retornados son la punta del iceberg de dichas políticas. Pero aún con todo ello, Sassen (2013, p. 15) nos recuerda que la ciudadanía se expandió "en parte gracias a las exigencias de los excluidos, tanto inmigrantes como ciudadanos, que no gozaban de plenos derechos". El trato al "otro", y en esto entran refugiados, solicitantes de asilo, 
exiliados, migrantes y retornados, es un medidor de lo avanzada que está una democracia.

\section{Conclusiones}

"El retorno tiene especificidades que obligan a repensar teóricamente el fenómeno" (Durand, 2006, p. 168). Entre dichas especificidades se encuentra que las relaciones familiares pueden variar dependiendo del éxito o fracaso, por ejemplo, si una de las motivaciones principales del mismo fue generar algún emprendimiento en el país de origen aprovechando el capital económico, educativo o cultural obtenido. Por otra parte, el retorno sirve para recordarnos que si bien toda cultura es de -y está en- cierto lugar, la identificación originaria de la misma se ha vuelto heterogénea. El estudio de las movilidad humana así lo demuestra.

No será lo mismo si el retorno es de uno de los miembros de la familia o si se trata del retorno de todo el núcleo familiar. Las decisiones acerca de quién migra, quién se queda y quién retorna afectan las relaciones e incluso los roles, lo cual a su vez implica decisiones en la crianza de los hijos, en el cuidado de los padres o abuelos, y demás. Se producen pues nuevas dinámicas familiares que pueden devenir separaciones, divorcios o desequilibrios emocionales pero también consolidación de lazos o asunción de mayores responsabilidades por parte de alguno de los miembros. Si uno de los proyectos migratorios es generar un ahorro con el fin de retornar al medio plazo, las decisiones acerca de quién migra primero - padre o madre- y qué empleo es capaz de conseguir, influirá en las relaciones familiares e incluso podrán darse, al mismo tiempo, procesos de empoderamiento, control o autonomía de uno o varios de los miembros del núcleo familiar (Parella y Cavalcanti, 2006). Concordamos con Stuart Hall (2003, p. 478), quien analizando las diásporas caribeñas afirma que "la familia extendida — como red y como lugar de memoria- es la principal mediación entre estos dos lugares [origen y destino]".

El fenómeno del retorno sirve para cuestionar el imaginario acerca de la familia nuclear tradicional, por cuanto muestra que más allá de la típica visión trinitaria padre-madre-hijo(s) son más bien las redes 
y las conexiones familiares las que muchas veces se tienen que hacer cargo de los cuidados y crianza. Las cadenas de afecto en este sentido desbordan la familia nuclear, por cuanto en ocasiones son hermanos, tíos, primos los que en etapas de la vida - muchas veces prolongadas por años- deben hacerse cargo de la manutención de los hijos de familiares o incluso del cuidado de personas mayores. En este sentido, los tradicionales roles de género de las mujeres también son puestos en cuestión, por cuanto son ellas las que muchas veces asumen el principal aporte a la economía, dado que en el país de destino, como en el retorno al de origen, son los principales agentes económicos. La construcción de género se realiza en este sentido también en una lógica transnacional, en donde el retorno puede ser el punto donde se afiancen los nuevos roles familiares.

Tras la migración, el lugar de origen ya no es la única fuente de identificación; lo que se refuerza y complejiza con el retorno. En este sentido, las políticas securitarias globales se hacen específicas en ciertas zonas del planeta, teniendo al sujeto migrante como eje principal de su accionar, lo cual, en el caso del retorno, se explicita principalmente en un enfoque ligado al economicismo, al emprendimiento y al desarrollo, los cuales se ven como positivos dentro de la lógica economicista clásica.

Compromisos, lazos, cambios en las expectativas y obligaciones alteran los objetivos de los migrantes e inciden en los tiempos y en la efectividad del retorno.

\section{Referencias bibliográficas}

Araujo, L. y Eguiguren, M. (2009). La gestión de la migración en los países andinos: entre la securitización y los vínculos diaspóricos. Sistema de Información sobre Migraciones Andinas, 3, 2-10.

Asamblea Nacional del Ecuador. (2017). Ley Orgánica de Movilidad Humana. Quito: Asamblea Nacional.

Bauman, Z. (2005). Vidas desperdiciadas. La modernidad y sus parias. Barcelona: Paidós.

Bauman, Z. (1999). La globalización. Consecuencias humanas. Ciudad de México: Fondo de Cultura Económica. 
Castillo, J. (1997). Teorías de la migración de retorno. Políticas de retorno de emigrantes. (s.d.).

Certeau, M. (1995). La Toma de la Palabra y Otros Escritos Políticos. Ciudad de México: Universidad Iberoamericana, Instituto Tecnológico y de Estudios Superiores de Occidente.

Cortés Maisonave, A. (2009). La migración ecuatoriana en el codesarrollo: elementos para una transnacionaldiad estatal. En Cortés, A. y Torres, A. (Coord.). Codesarrollo en los Andes: contextos y actores para una acción transnacional. Quito: Flacso.

Duque, G., y Genta, N. (2009). Crisis y migración: ¿el retorno esperado o búsqueda de alternativas? Boletín Andinamigrante, 5, 2-10.

Durand, J. (2006). Los inmigrantes también emigran: la migración de retorno como corolario del proceso. Revista Interdisciplinar da Mobilidade Humana, 14, (26-27), 167-189.

Hall, S. (2003). Pensando en la diáspora: en casa, desde el extranjero. Heterotopias: narrativas de identidad y alteridad Latinoamericana., 477-500.

López de Lera, D. (2010). Emigración, inmigración y retorno: tres etapas de un mismo proceso. Polígonos. Revista de geografía, 20, 9-27.

Martin, P. (2002). El espejismo de los trabajadores huésped mexicanos. Foreign Affairs: Latinoamérica, 2 (1), 204-220.

Mestries, F. (2013). Los migrantes de retorno ante un futuro incierto. Sociológica, 28 (78), 171-212.

Moreno, J. (2006). Feminización del fenómeno migratorio ecuatoriano. Historia Actual Online, 11, 121-132.

Moncayo, M. I. (2011). Políticas de retorno en América Latina: miradas cruzadas. Revista Andina Migrante. Dossier central.

Padrón Martínez, A. M. (2015). Análisis comparativo entre el plan bienvenidos a casa y el plan de retorno voluntario y su impacto en las relaciones internacionales entre Ecuador y España (Tesis).

Pagnotta, C. (2005). Ni aquí ni allá. L'immigrazione femminile dall'Ecuador. Alternativas. Cuadernos de Trabajo Social, 13.

Pajares, M. (2010) Inmigración y mercado laboral. Informe 2010. Madrid: Ministerio de Trabajo e Inmigración, Observatorio Permanente de la Migración 
Parella, S. y Cavalcanti, L. (2006). Una aproximación cualitativa a las remesas de los inmigrantes peruanos y ecuatorianos en España y a su impacto en los hogares transnacionales. Reis, 116, 241-257.

Pedone, C. (2013). Las representaciones sociales en torno a la inmigración ecuatoriana a España. Íconos-Revista de Ciencias Sociales, 14, 56-66.

Portes, A., Guarnizo, L., y Landolt, P. (2003). La globalización desde abajo: transnacionalismo inmigrante y desarrollo: la experiencia de Estados Unidos y América Latina. México: Flacso.

Ramírez, F. y Ramírez J. (2005). La estampida migratoria ecuatoriana. Crisis, redes transnacionales y repertorios de acción migratoria. Quito: Ediciones Abya-Yala.

Sassen, S. (2013). Inmigrantesy ciudadanos. De las migraciones masivas a la Europa fortaleza. Madrid: Siglo xxI.

Schramm, C. (2011). Retorno y reinserción de migrantes ecuatorianos. La importancia de las redes sociales transnacionales. Revista CIDOB d'Afers Internacional 92-93, 241-260.

Smith, M. P. y Guarnizo, L. E. (Eds.). (1998). Transnationalism from below. (Vol. 6). Transaction Publishers.

Villamar, D. y Acosta, A. (2002). Las remesas de los emigrantes y sus efectos en la economía ecuatoriana. Cartillas sobre Migración. Plan migración, Comunicación y Desarrollo, 1.

Waldinger, R. (2013). Más allá del transnacionalismo: una perspectiva alternativa de la conexión de los inmigrantes con su país de origen. Migraciones Internacionales, 7 (I), 189-219. 